\title{
La imagen de la psicología: EI Museo Gallego de Psicología
}

\author{
The image of Psychology: Galician Museum of Psychology
}

\author{
Alfredo Campos \\ Universidad de Santiago de Compostela
}

\begin{abstract}
Resumen
En esta comunicación efectuamos un recorrido histórico por el origen y desarrollo del Museo Gallego de Psicología de Galicia. Se analizan el origen de los distintos aparatos que posee el Museo, su creación y desarrollo hasta el momento actual. Se analizan también los fines que tiene el Museo, y puesto que está ubicado en la Universidad de Santiago de Compostela, su finalidad, además de recoger todo el material de psicología existente en Galicia para que no se pierda, se centra en el aspecto didáctico. La mayor parte de los aparatos están expuestos en vitrinas para que puedan ser vistos por los visitantes, y tienen, a su lado, una breve descripción de su finalidad y uso. En este trabajo también se exponen los planes de futuro del Museo, tanto en el plano didáctico como organizativo y de consecución de nuevos materiales. Pretendemos que el museo sea una imagen de la psicología.
\end{abstract}

Palabras clave: Psicología. Museo. Galicia. Imagen.

\section{Abstract}

In this paper we describe a historical journey through the source and development of the Galician Museum of Psychology. The origins of the different devices in the Museum, its creation and development to date are analysed. The aims of the Museum are also discussed, and since it is located at the University of Santiago de Compostela, its purpose, in addition to collecting all psychology related material existing in Galicia to avoid its loss, is focused on the didactic side. Most of the devices are exposed in showcases to allow the visitors to see them, and have displayed a brief description of its purpose and usage. In this paper the future plans for the Museum are also showed, in the educational and organizational levels and also in obtaining new materials. We intend that the museum be an image of psychology.

Keywords: Psychology. Museum. Galicia. Image.

\section{Antecedentes}

En Galicia, antes de 1970 había una Universidad, la Universidad de Santiago de Compostela, creada en el Siglo XVI por el Arzobispo D. Alonso de Fonseca y Acevedo, que obtiene del Papa Clemente VII, en el año 1526, una Bula por la que cede los derechos para crear los Grados para el Colegio de Santiago Alfeo, que él mismo había creado en el Hospital de la Azabachería, trasladados posteriormente al Palacio de Fonseca.

La Universidad de Santiago fue incrementando los estudios y extendiéndose, poco a poco, por Galicia a lo largo de los años (siglos). Uno de los impulsos más importantes de los últimos siglos de la Universidad de Santiago se produjo el 23 de Agosto de 1970, cuando fueron aprobados por el Gobierno los Estatutos provisionales de la Universidad de Santiago, y publicados, el 28 de Septiembre de 1970, en el Boletín Oficial del Estado. Al poner en práctica esta ley, se incorporaron a la Universidad de Santiago las Escuelas Universitarias y se crearon los Colegios Universitarios de A Coruña, Vigo, Ourense, y Lugo. Con la creación de los Colegios Universitarios se crearon también muchas titulaciones dependientes de la Universidad de Santiago de Compostela (Fuente: Universidad de Santiago de Compostela, 1978). A partir de este momento se incrementó mucho el número de profesores y de alumnos de la Universidad.

Hasta el año 1973 no se crearon en Galicia los estudios de psicología, por lo que, si se quería estudiar psicología, había que salir a estudiar fuera de Galicia. Con la aparición de los Colegios Universitarios se crearon los estudios de psicología en Galicia. Los estudios de la Licenciatura en Filosofía y Ciencias de la Educación, sección de Psicología (5 años académicos) estaban localizados en Santiago de Compostela. En los Colegios Universitarios de Ourense y A Coruña se crearon los tres primeros años de los estudios de Psicología, con la finalidad de que los alumnos fuesen estudiar el cuarto y quinto año a Santiago de Compostela, aunque también podías desplazarse a otra universidad.

Como la mayor parte del material que posee el museo proviene del Colegio Universitario de Ourense, por este motivo nos centramos a analizar la creación de su Laboratorio de Psicología.

\subsection{El Colegio Universitario de Ourense.}

El Colegio Universitario de Ourense fue creado mediante el Decreto de 18 de Agosto de 1972, publicado en el Boletín Oficial del Estado del 22 de Septiembre (B.O. E., $\mathrm{n}^{\mathrm{o}}$ 228), pero no comenzó a funcionar hasta 1973. El centro dependía económicamente de un Patronato, formado por la Diputación de Ourense, Caixa Ourense (hoy ABANCA) y otras instituciones locales. Académicamente dependía de la Universidad de Santiago. Esta seleccionaba el profesorado, imponía el plan de estudios, concedía títulos, etc. El Colegio Universitario de Ourense se instaló, en un primer momento, en un edificio de 8 plantas, propiedad Caixa Ourense (hoy ABANCA), en la Calle General Franco 35 (hoy, calle del Progreso).

El Laboratorio de Psicología del Colegio Universitario de Ourense se instaló en los bajos del edificio, a la llegada del profesor Alfredo Campos (Septiembre de 1977). Posteriormente (el 28 de Enero de 1989), el laboratorio se traslada a un nuevo edificio 
(Edificio de Hierro), situado en el recinto de un antiguo hospital, en la calle Doctor Temes, s/n. Este edificio fue donado por la Diputación de Ourense y el Ayuntamiento de Ourense a la Universidad de Santiago. Las instalaciones del nuevo Laboratorio de Psicología estaba formado por dos salas, unidas por cristales unidireccionales, y un almacén para los aparatos. Una de las salas era de experimentación, y la contigua, con gradas, era para la observación. Este laboratorio llegó a ser uno de los mejores de España en número de aparatos, y fue visitados por importantes personalidades de la psicología española.

El período durante el que el colegio Universitario de Ourense estuvo ligado a la Universidad de Santiago de Compostela fue desde el año 1972 hasta el año 1990. Mediante la aprobación de la Ley 11/1989, de 20 de Julio, de Ordenación del Sistema Universitario de Galicia (Diario Oficial de Galicia, 1989), y el posterior Decreto 3/1990, de 11 de Enero (Diario Oficial de Galicia, 1990), de Segregación de Centros y Servicios de la Universidad de Santiago de Compostela con todos sus medios materiales y humanos, y de su integración en las nuevas universidades de A Coruña y Vigo (Vaquero, 2001; Espada, 1995), el Colegio Universitario de Ourense pasó a depender de la Universidad de Vigo, y con él el Laboratorio de Psicología.

Al integrarse el Colegio Universitario de Ourense en la Universidad de Vigo, y dado que en esta Universidad no existía psicología, desapareció la Sección de Psicología de Ourense. Al desaparecer los estudios de psicología de Ourense, el Laboratorio de Psicología carecía de finalidad, por lo que comenzó un período de abandono y de desaparición del material.

\subsection{Plan de Estudios de Psicología del} Colegio Universitario de Ourense.

Pasamos ahora a comentar cuál era el Plan de Estudios de Psicología del Colegio Universitario de Ourense para comprender lo novedoso del Laboratorio de Psicología, teniendo un Plan de Estudios tan poco dado a la experimentación. Hay que decir, desde el principio que el Plan de Estudios del Colegio Universitario de Ourense era el mismo que el que tenía la Facultad en Santiago de Compostela.

Los estudios de psicología del Colegio Universitario de Ourense formaban parte de lo que se denominaba: "División de Filosofía y Ciencias de la Educación", que tenía el plan de estudios del año 1973. El Colegio Universitario tenía el mismo plan de estudios que la Facultad de Filosofía y Ciencias de la Educación de Santiago de Compostela. El Colegio Universitario de Ourense tenía sólo 3 cursos (los tres primeros), en cambio, la Facultad de Santiago tenía 5 cursos. El Colegio Universitario sólo tenía 2 especialidades: Psicología y Ciencias de la Educación, en cambio Santiago de Compostela tenía, además, la Sección de Filosofía.

En el Colegio Universitario de Ourense, los tres primeros cursos de la División de Filosofía y Ciencias de la Educación eran comunes (en el Plan de Estudios de 1973) para Psicología y Pedagogía (lo mismo que en Santiago de Compostela), si bien, en el segundo y tercer curso existía alguna asignatura específica de la especialidad.

El Plan de Estudios de Psicología (Plan de Estudios de 1973) era el siguiente:

Primer curso: Psicología General. Historia de la Filosofía Antigua y Medieval. Introducción Empírica a las Ciencias de la Educación. Lógica. Lengua Española.

Segundo curso (Comunes): Historia de la Filosofía Moderna y Contemporánea. Fundamentos Biológicos de la Personalidad. Antropología. Estadística Aplicada a las Ciencias de la Educación. Especialidad de Psicología: Psicodiagnóstico.

Tercer curso (Comunes): Ética. Sociología. Psicología Evolutiva. Especialidad de Psicología: Historia de la Psicología. Psicometría.

En el año 1979 cambia el Plan de Estudios de la Facultad de Filosofía y Ciencias de la Educación de Santiago de Compostela para que cada una de las divisiones tuviese una mayor especialidad, sin embargo, todavía se seguiría llamando Facultad de Filosofía y Ciencias de la Educación, con las Secciones de Filosofía, Pedagogía y Psicología. En el Colegio Universitario de Ourense se siguieron impartiendo los tres primeros cursos de las Secciones de Psicología y Pedagogía, pero ya con el nuevo Plan de Estudios. Los estudios de Psicología se convirtieron en Facultad de Psicología en la Universidad de Santiago de Compostela en el año 1991, pero en ese momento ya no existían estudios de Psicología en el Colegio Universitario de Ourense, que en ese momento dejó de llamarse Colegio Universitario para denominarse Campus Universitario de Ourense.

El Plan de Estudios de Psicología (Plan de Estudios de 1979) era el siguiente:

Primer curso: Psicología General I. Fundamentos Biológicos de la Conducta. Psicología Matemática I. Teoría y Métodos de la Psicología. Historia de la Filosofía Contemporánea en relación con la Psicología. Antropología.

Segundo curso: Psicología General II. Psicología Matemática II. Psicología Evolutiva I. Psicología Fisiológica I. Sociología.

Tercer curso: Psicología Fisiológica II. Psicología Evolutiva II. Psicología Experimental. Psicometría. Psicología Social. (Fuente: Universidad de Santiago de Compostela,1972, 1981, 1983).

\subsection{Utilidad del Laboratorio de Psicología del Colegio Universitario de Ourense. \\ El Laboratorio de Psicología del Colegio} Universitario de Ourense se ha creado con una doble finalidad: Con una finalidad docente y una finalidad investigadora.

El material del Laboratorio se utilizó, fundamentalmente, para impartir prácticas en las asignaturas de Psicología General e Historia de la Psicología (Plan de estudios de 1973). Con la implantación del Plan de Estudios de 1979 fue utilizado para las clases prácticas (hoy denominadas interactivas) de las asignaturas de Psicología General I, Psicología General II, y Psicología Experimental. Se procuraba que 
los alumnos, durante los tres años que pasaban en el Colegio Universitario, se familiarizasen con todos los aparatos, aprendiendo su manejo y utilidad. Además, con los aparatos más adecuados para el contenido de las asignaturas, los alumnos efectuaban réplicas de experimentos importantes de psicología. También se procuraba que la enseñanza de la psicología fuese lo más práctica posible, para contrarrestar que la carrera estaba prevista como algo bastante teórico.

En esta época existían pocos manuales de prácticas de psicología, por lo que el profesor Alfredo Campos publicó, en el año 1988, un libro: "Manual de prácticas de psicología básica". Barcelona: Grupo Editor Universitario. El libro estuvo de manual de prácticas en muchas universidades españolas y latinoamericanas.

El libro consta de 18 capítulos que abordan los temas fundamentales de la psicología básica: 1.- El experimento psicológico. 2.- Percepción de profundidad. 3.- Percepción del tiempo. 4.- Percepción de figuras. 5.- Ilusiones ópticas. 6.- Posefectos. 7.Memoria. 8.- Olvido. 9.- Imágenes mentales. 10.Formación de conceptos. 11.- Resolución de problemas. 12.- Condicionamiento clásico. 13.- Condicionamiento instrumental. 14.- Refuerzo. 15.- Transferencia. 16.Motivación. 17.- Frustración y conflicto. 18.Emociones.

La forma de efectuar las prácticas era bastante innovadora para esa época. Se procuraba hacer réplica de experimentos importantes del contenido de las asignaturas. Los alumnos, en grupos de 4 ó 5, elegían una práctica y el aprendían con el profesor la forma de llevarla a cabo y posteriormente ellos la dirigían a todos los compañeros, en pequeños grupos. Es de esta forma cada alumno tenía que impartir varias horas de clase.

En cada práctica se hacía una réplica real de un experimento importante de psicología (recogiendo los datos de los compañeros, en la hora de la práctica), y analizando el grupo los resultados. También se especificaban todos los pasos del experimento: Título, problema, hipótesis, variable independiente y dependiente, grupo control y experimental, etc. (McGuigan, 1974). Si la práctica se efectuaba con animales (ratas, gatos, peces...), y el tiempo dedicado a la práctica no era suficiente para llevarla a cabo, el grupo que dirigía la práctica efectuaba el experimento fuera de las horas lectivas $\mathrm{y}$, posteriormente, en el horario de las prácticas, hacía una demostración con los animales, y comunicaba los resultados.

Los alumnos que dirigían la práctica también debían hacer un informe que, posteriormente, estaba a disposición de todos los compañeros para que sobre él, hiciesen un resumen. Todos los alumnos debían hacer un resumen de todas las prácticas. Este resumen tenía un valor para la calificación final. La asistencia a las prácticas era obligatoria.

Algún material del laboratorio era utilizado por los profesores o investigadores para hacer sus investigaciones. Tenemos que tener en cuenta que era material muy preciso y no existían, todavía los ordenadores.

\section{El Museo Gallego de Psicología: Los comienzos.}

\section{A) Finalidad.}

Existen varias razones que nos llevaron a crear el Museo Gallego de Psicología:

1.- Había mucho material de psicología que no se utilizaba en la Universidad de Santiago de Compostela.

2.- Había mucho material de psicología que no se utilizada en las Universidades de A Coruña y Vigo.

3.- El material de psicología que está en desuso, se tira a la basura, con la consiguiente pérdida de cultura, que, una vez perdida ya es irrecuperable. El patrimonio de las universidades se estaba tirando.

4.- El material se podía salvar de la basura, y así salvar un patrimonio.

5.- Con el material de psicología existente en Galicia (y también fuera de Galicia) se podía crear un Museo de Psicología de Galicia.

6.- El Museo tiene una finalidad didáctica para los alumnos del grado, para los profesores y para los visitantes. Todos ellos pueden ver los aparatos y adquirir unos conocimientos básicos sobre cada uno de los aparatos, pues cada aparato tiene una tarjeta indicando su funcionamiento y utilidad.

\section{B) Ubicación.}

El material del Museo de Psicología de Galicia se encuentra ubicado, de momento, en unos expositores que se encuentran en los pasillos de la Facultad de Psicología de la Universidad de Santiago de Compostela. Se eligió esta ubicación porque, de este modo el material está siempre visible para los alumnos, y para cualquier persona

Los expositores se han diseñado teniendo en cuenta el material que iban a albergar. Existen dos expositores que están en la planta de entrada de la Facultad que tienen, desde mi punto de vista, los instrumentos más importantes de psicología a lo largo de todos los tiempos. Un expositor tiene la Caja de Skinner, y el otro expositor tiene una taquistoscopio de dos canales. Otros expositores tienen uno o dos estantes, en función del material que se expone.

Los expositores tienen dos partes, la parte superior es toda de cristal resistente, para que se puedan ver los aparatos desde todos los niveles. La parte de debajo de los expositores es de madera con dos puertas. Tiene una altura de $90 \mathrm{cms}$. Se construyó de este modo para que los aparatos queden altos para facilitar su visión, y la parte baja sirva como almacén.

\section{C) EI logotipo.}

El logotipo del Museo Gallego de Psicología es obra de la profesora y artista Maricarmen Torres Maroño. Es una $\psi$ griega, símbolo de Psicología. La "Psi" está formada por distintos colores: El amarillo por la relación de la psicología con la sanidad, el morado que es el color del traje académico de la psicología en la Universidad, y del color azul que representa la relación de la psicología con la enseñanza. Bordeando la letra "Psi" un letreo en español y gallego que dice: "Museo 
Gallego de Psicología", y "Museo Galego de Psicoloxía".

\section{D) Procedencia del material.}

El material del que consta el museo procede, fundamentalmente, de la Universidad de Vigo (antiguo Colegio Universitario de Ourense), en segundo lugar, de la Universidad de Santiago de Compostela, y en mucha menos medida de la Universidad de A Coruña (antiguo Colegio Universitario de A Coruña). También hubo donaciones de personas particulares. En estos momentos existen en el Museo alrededor de sesenta aparatos, pero existen muchos más aparatos localizados en distintas universidades gallegas, que se tratará de que sean depositados en el Museo para incrementar su número, y que no desaparezcan.

El material del museo fue construido por distintas empresas de diferentes países: Gerbans Corporation, Letica, ASI Instruments, Kodak, Eumig, Campden, Mepsa, Lafayette, Taki-Co Ltd. Algunos aparatos fueron diseñados por nosotros y construidos en Galicia.

\section{E) EI libro de registro.}

Hemos diseñado y mandado confeccionar un Libro de Registro. Este Libro tiene las siguientes columnas: Número de Registro, Fecha, Nombre del Material, Procedencia, Observaciones. El Libro de Registro fue, a su vez, registrado en la Facultad de Psicología el día 24 de Julio de 2014, con el número 72. En el Libro de Registro está anotado todo el material que va llegando al Museo.

\section{F) La inauguración.}

El Museo de Psicología de Galicia fue inaugurado el día 24 de Febrero de 2012, día de la fiesta del Patrón de la Facultad (Juan Huarte de San Juan), por la Decana de la Facultad de Psicología, la Profesora Carolina Tinajero Vacas. El día 19 de Mayo de 2014 fue elegido por la Junta de la Facultad de Psicología de la Universidad de Santiago de Compostela como primer Director del Museo Gallego de Psicología, el Profesor Alfredo Campos García.

\section{Presente y futuro.}

El Museo es muy joven, y si bien todos los museos están constante cambio, éste, al ser tan joven, necesita consolidarse y conseguir un mínimo de infraestructura. Tiene un amplio programa didáctico y un detallado programa de futuro para poder recuperar una gran cantidad de material que todavía no se encuentra en el Museo, y extenderlo con mayor profundidad a los alumnos y a la sociedad.

\section{A) Actividad didáctica.}

El Museo se ha creado con una finalidad didáctica. Las principales actividades didácticas son las siguientes:

1.- Se procura que todos los aparatos estén visibles para los alumnos para que se vayan, poco a poco, familiarizando con los instrumentos.

2.- Se organizan todos los años visitas guiadas para los alumnos del primer curso del Grado en Psicología.
3.- En la Fiesta de Huarte de San Juan, Patrón de la Facultad, se organizan visitas guiadas para los profesores.

4.- Se coopera con otros museos. Por ejemplo, este curso se ha colaborado con MARCO (Museo de Arte Contemporáneo de Vigo), en una exposición temporal, al que se le han prestado varios aparatos relacionados con la percepción.

\section{B) Perspectiva de futuro.}

El Museo tiene una serie de retos que alcanzar en los próximos años para consolidarse como un auténtico museo. Entre otros, son los siguientes:

1.- Conseguir más expositores, los que hay están repletos de material.

2.- Conseguir más material. Tenemos localizado una cantidad grande de aparatos que si no conseguimos traerlos y registrarlos en el Museo, corren el riesgo de perderse como sucedió con tanto material.

3.- Conseguir unos locales para almacenar mucho material que no caben en los expositores, sobre todo material de grandes dimensiones.

4.- Confeccionar una página web en la que se exponga todo el material existente, con comentarios de cada uno de los aparatos. Esto dará difusión al Museo.

5.- Extender el Museo también a los test. De momento en el Museo sólo existe material de laboratorio.

6.- Efectuar un programa detallado de actividades, tanto para los alumnos de la Universidad, como para personas de fuera de la Universidad, en la que se hagan jornadas didácticas sobre la historia y utilizad de todo el material del Museo.

\section{Esquema de clasificación del material del Laboratorio}

En la clasificación del material del Museo hemos seguido la clasificación de MEPSA (principal casa distribuidora española de material de psicología) en sus catálogos (MEPSA, 1978). MEPSA clasificaba el material de psicología en los siguientes apartados: A) Material de enseñanza e investigación: Tiempos de reacción, memoria, taquistoscopios y otros fenómenos perceptivos, fisiología general, psicología y fisiología animal, varios. B) Material de diagnóstico psicológico y físico: Aptitudes, dinamometría, psicofisiología deportiva, sensoriometría. C) Material de terapia: Lenguaje, psicoterapia, bioinformación. Esta misma clasificación será la que se pondrá en la página web del Museo, que estará unida a la Facultad de Psicología de la Universidad de Santiago.

\section{Referencias}

Bernal, M., Sampedro, M. J., Alcaraz, M., Blanco, M., Campos, A., Leirós, L., y Méndez, C. (2012). Libro de actas del XXV Symposium de la SEHP. A Coruña: Imprenta Provincial de A Coruña.

Boletín Oficial del Estado (1972, $\mathrm{n}^{\circ}$. 228).

Campos, A. (1988). Manual de prácticas de psicología básica. Barcelona: Grupo Editor Universitario.

Campos, A. (2012). El laboratorio de psicología del Colegio Universitario de Ourense (1972-1990): 


\section{EL MUSEO GALLEGO DE PSICOLOGÍA}

Pionero en España. Boletín de la Sociedad Española de Historia de la Psicología, 49, 2-10.

Diario Oficial de Galicia (1989), $\mathrm{n}^{\mathrm{o}} 156$, de 16 de agosto.

Diario Oficial de Galicia (1990), n ${ }^{\circ} 20$, de 24 de Enero.

Espada, L. (1995). La Universidad de Vigo: Antecedentes y etapa constituyente (1988-1994). Vigo: Fundación Provigo.

González Lorenzo, M., y Romaní Martínez, L. (Eds.) (1990). Universidad en Orense. Presente y Futuro. Confederación Empresarial Orensana.

Lafayette, Instrument Co. (1977). A catalog of scientific and laboratory instruments for the life sciences. Indiana: Lafayette.

Lafayette, Instrument Co. (1980). General psychological. Vol 1.Indiana: Lafayette.

Lafayette, Instrument Co. (1980). Timing, programming \& data collection. Vol 2). Indiana: Lafayette.

Lafayette, Instrument Co. (1980). Animal behavior systems \& supplies. Vol 3). Indiana: Lafayette.

Lafayette, Instrument Co. (1984). General psychological. Vol 1. (1984-85). Indiana: Lafayette.

Lafayette, Instrument Co. (1990). Lafayette instrument 1990-91 catalog. Indiana: Lafayette.

Lafayette, Instrument Co. (1992). Psychology/biology catalog (1992-93). Indiana: Lafayette.
McGuigan, F. J. (1974). Psicología experimental. México: Trillas. MEPSA (1978). Catálogo general. Madrid: MEPSA.

Plataforma Pro Campus (1995). Universidade de Ourense 1995. Mapa de titulacións. Ourense: Ayuntamiento de Ourense.

SYMTEC (2012). Catálogo 2012. Madrid: SYMTEC.

Vaquero, A. (2001). El proceso de segregación universitaria en Galicia: El caso particular del Campus de Ourense. En J. Gómez (Ed.). X Jornadas de la Asociación de Economía de la Educación (pp. 157-168). Murcia: Servicio de Publicaciones de la Universidad de Murcia.

Universidad de Santiago de Compostela (1978). Guía Académica. Curso 1977-78. Santiago de Compostela: Servicio de Publicaciones de la Universidad de Santiago de Compostela.

Universidad de Santiago de Compostela (1981). Guía Académica. Curso 1981-82. Santiago de Compostela: Servicio de Publicaciones de la Universidad de Santiago de Compostela.

Universidad de Santiago de Compostela (1983). Guía Académica. Curso 1983-84. Santiago de Compostela: Servicio de Publicaciones de la Universidad de Santiago de Compostela. 